\title{
Second generation of vortex-antivortex states in mesoscopic superconductors: Stabilization by artificial pinning
}

\author{
R. Geurts, M. V. Milošević, and F. M. Peeters* \\ Departement Fysica, Universiteit Antwerpen, Groenenborgerlaan 171, B-2020 Antwerpen, Belgium
}

(Received 2 February 2009; revised manuscript received 3 April 2009; published 6 May 2009)

\begin{abstract}
Antagonistic symmetries of superconducting polygons and their induced multivortex states in a homogeneous magnetic field may lead to the appearance of antivortices in the vicinity of the superconducting/normalstate boundary (where mesoscopic confinement is particularly strong). Resulting vortex-antivortex (V-Av) molecules match the sample symmetry but are extremely sensitive to defects and fluctuations and remain undetected experimentally. Here we show that V-Av states can reappear deep in the superconducting state due to an array of perforations in a polygonal setting, surrounding a central hole. Such states are no longer caused by the symmetry of the sample but rather by pinning itself, which prevents the vortex-antivortex annihilation. As a result, even micron size, clearly spaced $V$-Av molecules can be stabilized in large mesoscopic samples.
\end{abstract}

DOI: 10.1103/PhysRevB.79.174508

PACS number(s): 74.78.Na, 74.25.Dw, 74.25.Qt

Vortices in small superconducting elements have been the focus of scientific research ever since it was shown that a strongly confined superconducting condensate shows enhanced critical properties. ${ }^{1-3}$ Usually individual vortices pierce the sample in the direction of applied magnetic field, minimizing the energy of the stray magnetic field, as well as their mutual interaction. Such formed vortex clusters (called "multivortex") tend to mimic the symmetry of the sample due to the repulsion with the surrounding screening (Meissner) currents. In samples on the order of the coherence length $\xi$ and/or penetration depth $\lambda$, the edge currents may even compress the vortex lines into a single bundle, often referred to as a "giant vortex." ${ }^{4,5}$ However, in addition to these simple arguments, the effects of lateral confinement on mesoscopic elements can have more elaborate manifestations. One such is the appearance of vortex-antivortex ( $\mathrm{V}-\mathrm{Av})$ molecules of the same symmetry as the host sample.

Almost a decade since the original prediction of Chibotaru et al. $^{6}$ the symmetry-induced antivortex remains an exciting phenomenon in vortex matter in submicron superconductors. The stability of an antivortex in opposite magnetic field is still puzzling, and not yet verified experimentally. The latter is not surprising, taking into account that the predicted vortex-antivortex molecules are very small in size $\left(\sim \xi\right.$, somewhat larger in type-I samples $\left.{ }^{7}\right)$ and extremely sensitive to defects. ${ }^{8}$ Actually, in most instances, they are simply unstable. ${ }^{9,10}$

In the last several years, two methods were proposed to improve the stability and the observability of the vortexantivortex states in mesoscopic polygons. In one, the magnetic field profile was altered by a magnetic dot placed on top of the superconductor. ${ }^{11}$ The bipolar field of the added magnet favors the antivortex underneath and repels vortices further apart. However, this method: (i) interferes with the key concept of an antivortex in opposite unipolar field, (ii) makes the structure more complicated, three-dimensional, and inaccessible for scanning probe techniques, and (iii) poses difficulties for any magnetic (magnetic force, Hallprobe) measurements. Instead, we recently proposed the structural engineering of the sample itself, by strategically placed holes in the sample, mimicking the sample symmetry and the expected symmetry of the vortex-antivortex state. ${ }^{12}$ In such realization each hole hosts (pins) one vortex, where the pinning force is effectively stronger than the vortexantivortex attraction. As a consequence, vortices remain in the holes even when placed at further distances from the antivortex, and the whole molecule can be made significantly larger.

In above works, the "first generation" of V-Av states was predicted theoretically. Such states in a unipolar magnetic field were always found as a consequence of the symmetry of the sample. Here we present the second generation of $\mathrm{V}-\mathrm{Av}$ states, found deeper in the superconducting state, and with different physical background. With respect to Ref. 12, we realized that not only vortices can be pinned using the latter concept but also an antivortex as well. We therefore introduced an additional, central hole in the sample, in which the antivortex can reside. Intuitively, this facilitates the quantization of negative-stray flux between the vortices in a cluster, and leads to a more stable antivortex. Vortices and antivortices in holes are just quantized magnetic field, screening prevents their annihilation, and the fact that they have no core reduces their attractive interaction. As will be shown, this indeed leads to an enhanced stabilization of the vortexantivortex molecule, even deep into the superconducting state, but with significantly altered overall properties. Although this generally applies to any polygonal setting of the holes and various shapes of the sample, ${ }^{13}$ in what follows we show the proof of principle for a square superconducting geometry, as shown in Fig. 1.

For the purpose of this study, we use the mean-field Ginzburg-Landau (GL) theory and solve numerically the set of GL equations

$$
\begin{gathered}
(-i \nabla-\vec{A})^{2} \psi-\left(1-T-|\psi|^{2}\right) \psi=0, \\
-\kappa^{2} \Delta \vec{A}=\vec{j}_{s}=\Re\left[\psi(i \nabla-\vec{A}) \psi^{*}\right],
\end{gathered}
$$

already established as a prime tool for theoretical description of mesoscopic superconductors. ${ }^{4}$ All variables in Eqs. (1) and (2) are dimensionless and expressed in temperatureindependent units [assuming $\sim(1-T)^{-1 / 2}$ dependence of 


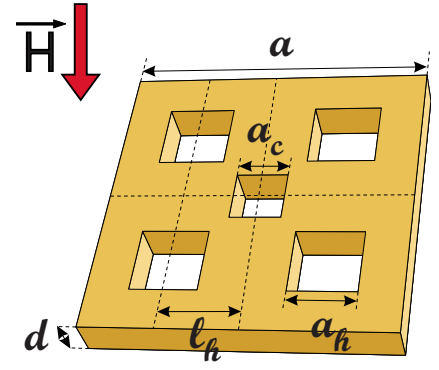

FIG. 1. (Color online) A schematic diagram of a polygonal mesoscopic superconductor (side $a$, thickness $d$ ) with corresponding arrangement of perforations (spacing $l_{h}$, characteristic size of each hole $a_{h}$, and of the central hole $a_{c}$ ) in a perpendicular magnetic field $H$.

characteristic length scales in the system, coherence length $\xi$, and penetration depth $\lambda] . \kappa=\lambda / \xi$ is the so-called GL parameter and describes the ability of the sample to screen the applied magnetic field. Temperature is scaled to $T_{c}$, magnetic field to $H_{c 2}(0)$, vector potential to $H_{c 2}(0) \xi(0)$, and the order parameter $\psi$ to $\psi(0)=\sqrt{\alpha(0) / \beta}$. The Gibbs free energy density $\mathcal{F}$, in units of $\mathcal{F}(T=0, H=0)=H_{c}(0)^{2} / 8 \pi$, is calculated from

$$
\begin{aligned}
\mathcal{F}= & \frac{1}{V} \int\left[|(-i \nabla-\vec{A}) \psi|^{2}-\left(1-T-\frac{1}{2}|\psi|^{2}\right)|\psi|^{2}\right. \\
& \left.+\kappa^{2}(\nabla \times \vec{A}-\vec{H})^{2}\right] d V,
\end{aligned}
$$

for each of the stable superconducting states found during the simulation. The full simulation region, used for the calculation of the magnetic response of the sample, was a square with 128 grid points in each direction. The number of grid points inside the sample was kept at $64 \times 64$.

In Fig. 2 we show the free energy of vortex states with vorticities from $L=0$ to $L=6$ as a function of the applied magnetic flux $\Phi=a^{2} H$. The simulation is performed by sweeping the magnetic field up and down, and then backtracking of all found vortex states so that their complete stability range is obtained. In addition, we performed the calculation starting from different initial conditions, some of which included reasonable guesses of possible vortex configurations. Figure 2 summarizes all the found stable states, but we may not rule out the possibility of some more complex, higher energy, vortex states. For this simulation a square of size $20 \xi(0) \times 20 \xi(0)$ was used with holes of size $3.5 \xi(0) \times 3.5 \xi(0)$, four of which are horizontally and vertically displaced by $5 \xi(0)$ from the center of the sample. The temperature was fixed at $0.3 T_{c}$ [corresponding to Al samples below $400 \mathrm{mK}$ (Ref. 14)] and an extreme type-II behavior was assumed, typical for thin samples $(\kappa=\infty)$. The combinatorial number of possible vortex configurations for given vorticity is quite high, and many of them are indeed found stable due to both the large size of the sample and that of the central hole. Starting from zero field, the Meissner state, $L$ $=0$, is the lowest-energy state in the flux band of $\Delta \Phi$ $=1.85 \Phi_{0}$, which is larger than a flux quantum but significantly smaller than in nonperforated mesoscopic samples. ${ }^{10}$ The reason is that the first vortex penetrates easier and is more stable in the present system due to the central hole (central $L=1$ is in the ground state in $\Delta \Phi=1.92 \Phi_{0}$ ). States with an off-center vortex have mostly higher energy, smaller stability range, and are fourfold degenerate (which makes them interesting for logic applications ${ }^{15}$ ). $L=2$ shows the broken symmetry in the ground state, as two vortices reside in the central and corner hole, but their ground-state flux band is only $\Delta \Phi=0.17 \Phi_{0}$. Similarly, the broken symmetry $L=3$ state with all vortices in the corners is the ground state

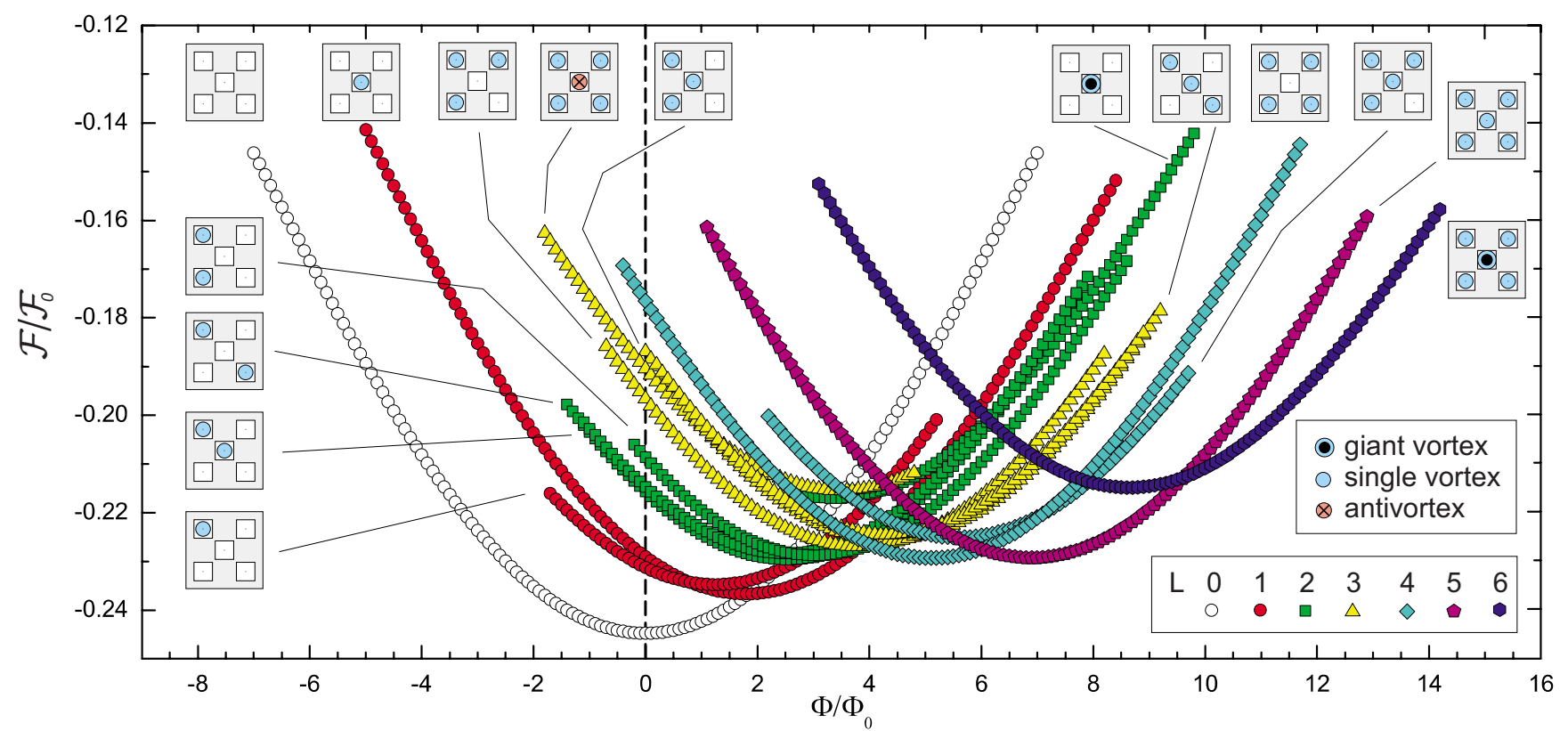

FIG. 2. (Color online) The Gibbs free energy spectrum, showing the energy levels and stability of different vortex states as a function of applied magnetic field at $T=0.3 T_{c}$. Insets show schematics of the vortex arrangement for selected states. 


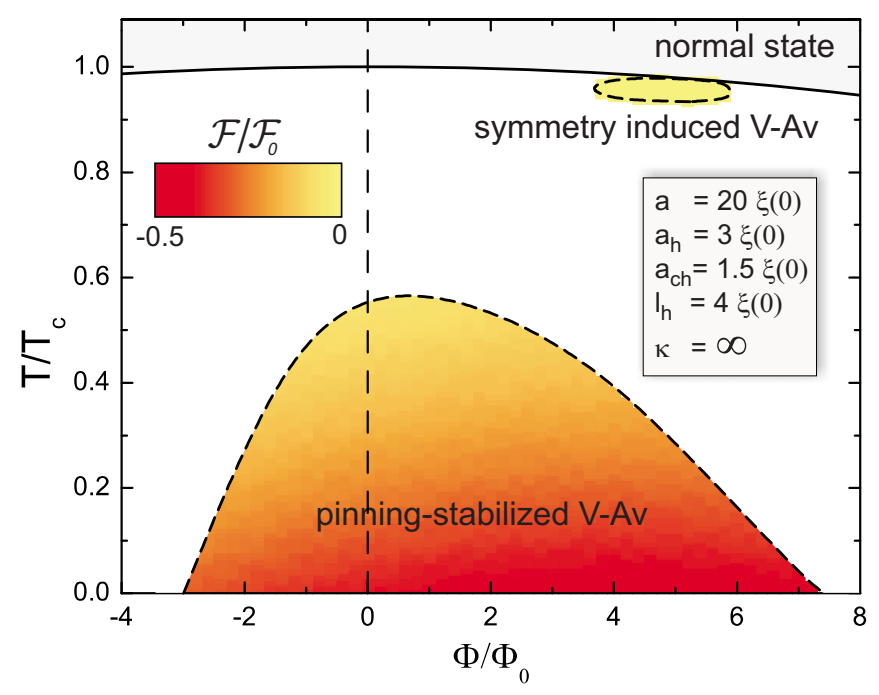

FIG. 3. (Color online) The superconducting state in the $\Phi-T$ diagram (solid line shows the superconducting/normal-phase boundary) comprising the areas of stability of the $L=3$ vortexantivortex molecules, both pinning- and symmetry-induced ones. Color coding shows the energy of those vortex states.

in a short interval of $\Delta \Phi=0.11 \Phi_{0}$. Just like in the superconducting square with $2 \times 2$ holes, ${ }^{16} L=4$ vortices reside in the corners and show enhanced stability in the ground state $\left(\Delta \Phi=1.95 \Phi_{0}\right)$. However, the most dominant state in Fig. 2 is the $L=5$ one, where all holes are occupied by a single vortex $\left(\Delta \Phi=3.63 \Phi_{0}\right)$. Therefore, while for the square with $2 \times 2$ holes high-symmetry states, such as $L=2$ and $L=4$, were pronouncedly low-energy states, we notice that in the present system the states which have a vortex in the central hole, and are fourfold symmetric, dominate the ground state, i.e., $L$ $=1$ and $L=5$. Note that the $L=4$ state is still very comfortable with the fourfold symmetry although it does not have a central vortex, but, e.g., $L=3$ with a central vortex is not in the ground state because it does not obey the symmetry of the sample. Because of their very low energy, $L=1,4,5$ states overshadow other states such as $L=2$ and $L=3$. The $L=3$ state has several allotropes; i.e., it can have vortices across the sample diagonal, one vortex in the center and two on the side, or all three out of the center. Only one configuration can satisfy the fourfold symmetry of the sample and that is the $L=4-1$ state, with four vortices in corner holes and an antivortex in the center. This $\mathrm{V}-\mathrm{Av}$ state is indeed found stable in our system, with relatively high energy, but still lower than most of the higher-vorticity states (which suggests that it can be experimentally realized in a decreasing magnetic field).

The physical origin of this V-Av state is, however, different from the V-Av state found in Refs. 6-8 and 12 where the $\mathrm{V}$-Av molecules appear due to the imposed symmetry of the sample. In Ref. 12, the added holes only reinforce the symmetry argument and do not cause per se the V-Av state. In the rest of the paper, we will refer to this state as the symmetry-induced $\mathrm{V}-\mathrm{Av}$, in contrast to the here reported $\mathrm{V}-\mathrm{Av}$ state which is fully stabilized by pinning all vortices and the antivortex. In Fig. 3 we show the $\Phi-T$ phase diagram for both versions of the $L=3$ vortex-antivortex state (for the parameters $a=20 \xi(0), a_{h}=3 \xi(0), l_{h}=4 \xi(0)$, and $a_{c}=1.5 \xi(0)$, see Fig. 1). We observed two fully independent islands in the $\Phi-T$ phase space corresponding to the two manifestations of the V-Av state. The symmetry-induced one is situated in the high-temperature regime, where the confinement imposed by the sample boundaries is effectively very large. It should be noted here that the $\Delta T$ stability range for the symmetryinduced $\mathrm{V}$-Av state is on average just $1 \%$ larger than in the case of $2 \times 2$ holes $^{12}$ (since the order parameter inside the molecule is in any case severely suppressed). At lower temperatures, the GL equations are strongly nonlinear and symmetry arguments cannot account for the nucleation of the $\mathrm{V}-\mathrm{Av}$ state. Nevertheless, a second-generation V-Av state does stabilize, thanks to the large spacing between the holes (preventing vortex-antivortex annihilation) and the large size of the sample (diminishing the effect of encircling screening currents). The pinning-stabilized V-Av state: (1) occupies far larger $\Phi-T$ area compared to the symmetry induced one, a wide region of $\approx 10 \Phi_{0}$ and $\approx 0.6 T_{c}$ and (2) it is found stable even in a negative applied field, where central antivortex is a natural state and vortices are subjected to an increasing expulsion pressure. In principle, several vortex states are found stable at negative fields in Fig. 2, which is a manifestation of the flux-trapping effect. ${ }^{17}$ However, out of all $L=3$ states, $L=4-1$ shows maximal resilience to negative flux.

To emphasize again, the size of the sample plays a crucial role for the V-Av state. While the symmetry-induced V-Av exists only for small samples [compared to the coherence length $\xi(T)$ ] the pinning-stabilized $\mathrm{V}$-Av requires a large sample. This is illustrated in Fig. 4(a) for a square with five holes. When the sample is larger, the flux-stability region of the pinning-stabilized V-Av state becomes larger as well. We can attribute this to the weakened influence of the Meissner current on the inside vortices because of the larger distance. One also notices the extremely large flux interval (from $-7.5 \Phi_{0}$ to $17 \Phi_{0}$ ) for stability of the $L=3$ pinning-stabilized $\mathrm{V}$-Av state. However, the penetration field for new vortices must decrease for larger samples (see e.g., Ref. 18 for the case of large BiSCCO disks). It is already known that a magnetic field higher than $H_{c 1}$ is needed for the penetration of vortices into mesoscopic superconductors, but this factor decreases to unity in bulk systems. Nevertheless, the latter factor decreases with the size of the sample much slower than the square power increase of the area of the sample. As a consequence, the threshold flux for penetration of new vortices in our sample increases as a function of $a / \xi(0)$. We also observed the changing curvature of the latter dependence [at $a / \xi(0) \approx 25$ ]; from the calculation of the threshold magnetic field, we found that samples with size of $10-25 \xi(0)$ exhibit almost identical demagnetization effects, i.e., similar vortex penetration field which then gradually decreases for sizes above $25 \xi(0)$.

The size of the central hole is equally important as the sample size, as it relates to the ability of V-Av pairs to annihilate. Consequently, this second-generation V-Av state cannot be stabilized in the absence of the central hole [see Fig. 4(b)]. The effect of decreasing the central hole size is also indicated in Fig. 4(b) - the upper boundary of the flux stability interval decreases due to the facilitated V-Av annihilation (at high fields, vortices are pushed toward the center of the 

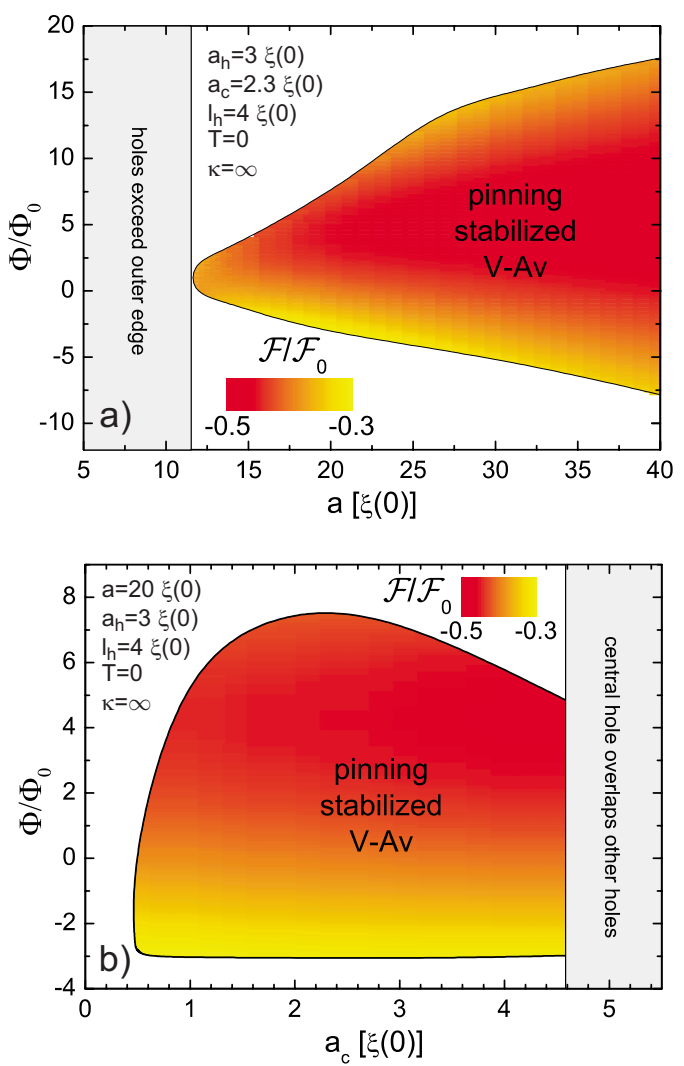

FIG. 4. (Color online) The stability diagram versus applied field (i.e., magnetic flux) for the pinning-induced vortex-antivortex state (a) as a function of the sample size for a fixed temperature and (b) as a function of the size of the central hole.

sample), while the lower boundary is in fact not influenced by the size of the central hole. Note that the lower boundary lies in the negative-field region, where vortices have an expulsion tendency, and pinning of any strength easily stabilizes the central antivortex.

The latter stability of the pinned V-Av state in the negative field forms the base for realizing this state in experiments. Namely, an abrupt increase of the applied magnetic field from a low negative value (that stabilizes one antivortex in the sample) to a large positive one (allowing the penetration of multiple vortices in outer holes) may result in the desired V-Av state at low temperatures. Alternatively, following the results of Fig. 3, one can think of an abrupt temperature decrease. We performed a corresponding simulation, where we started from the symmetry-induced V-Av state (which is the ground state for $L=3$ at temperatures near $T_{c}$ ) and then applied a steep temperature decrease. Without taking into account the experimentally relevant temperature gradient over time, the simulations were always able to land into the pinning-stabilized V-Av state at low temperatures. This is illustrated in Fig. 5 where also the influence of $\kappa$ is depicted. Stronger screening of the magnetic field into the holes (i.e., lower $\kappa$ ) enhances the pinning, and consequently favors the $\mathrm{V}$-Av state of second generation, in contrast to the symmetry-induced V-Av which is disfavored by low values of $\kappa$ due to the effective attraction of vortices into a single bundle (see Ref. 12).

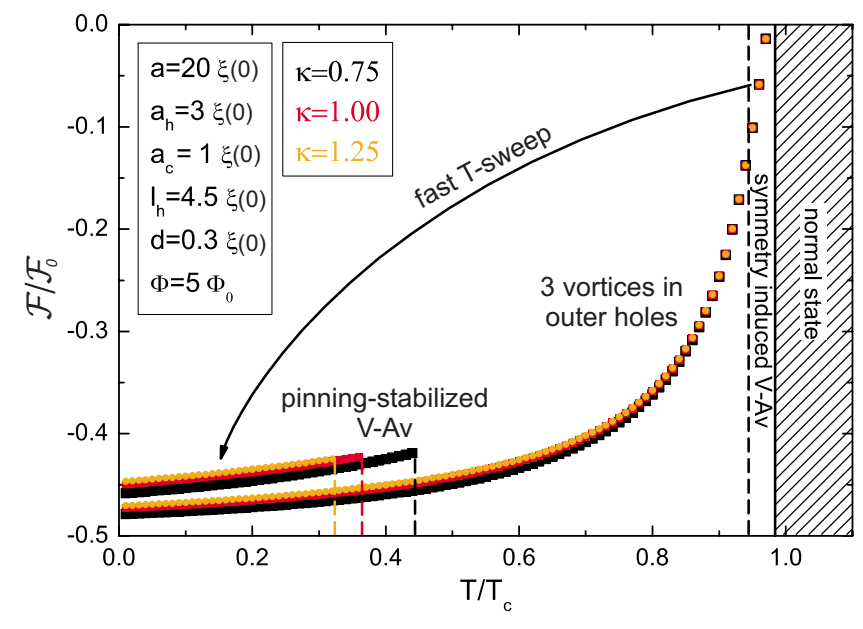

FIG. 5. (Color online) The energy of V-Av molecules as a function of temperature for different values of the Ginzburg-Landau parameter $\kappa$. In numerical simulations, an abrupt cooling enables a direct transition between two kinds of V-Av states.

Although quite difficult to realize (never being the ground state of the system), the second-generation V-Av state is much more suitable for direct experimental visualization than its symmetry-induced ancestor. First, samples can be made larger, and spacing between vortices and the antivortex are sufficient for their identification. Second, the Cooper-pair density is significantly higher inside the V-Av molecule since temperature may be far below $T_{c}$. Third, the magnetic field profile in the sample is sufficiently inhomogeneous, with pronounced amplitudes, benefiting from the size of the $\mathrm{V}-\mathrm{Av}$ state and its stability for low values of $\kappa$. This is shown in Fig. 6 where the magnetic field profile in and nearby the sample is calculated for two values of $\kappa$ for the secondgeneration $\mathrm{V}-\mathrm{Av}$ and for one value for the symmetry-induced $\mathrm{V}$-Av (given in real units). For the parameters selected here, the magnetic field contrast inside the symmetry-induced
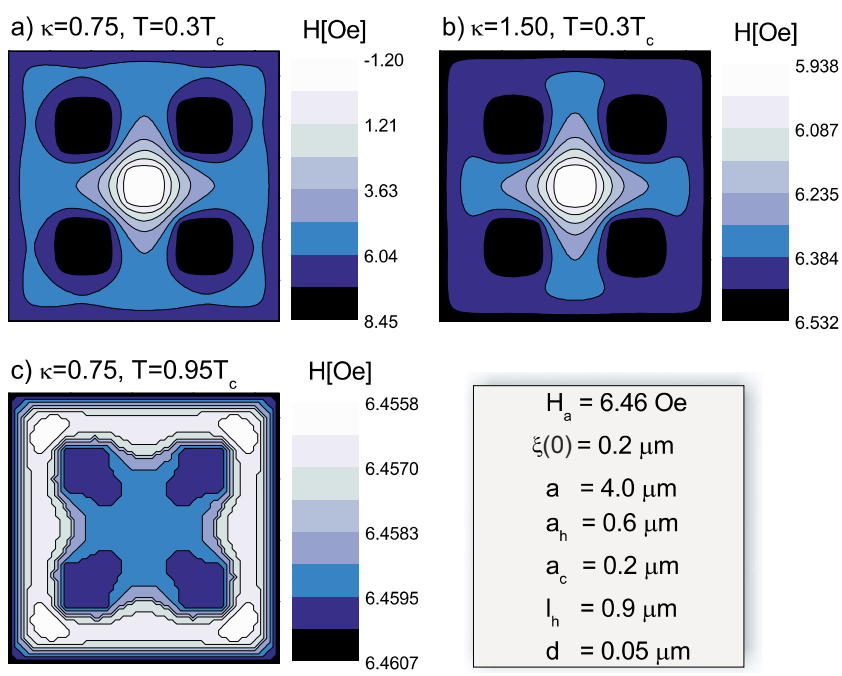

FIG. 6. (Color online) Calculated magnetic field profiles in the sample: (a) for the pinning-stabilized V-Av state (b) two values of $\kappa$, and (c) the corresponding profile for the symmetry-induced $\mathrm{V}-\mathrm{Av}$ state. 
$\mathrm{V}$-Av molecule is of the order of $0.01 \mathrm{Oe}$, while that of the second-generation $\mathrm{V}$-Av can be above $10 \mathrm{Oe}$, thus 3 orders of magnitude larger.

Using a square superconducting geometry with five holes, we found a second generation of the $L=3 \mathrm{~V}-\mathrm{Av}$ state in a uniform field. This state is purely induced by pinning, and is not caused by the symmetry of the sample, contrary to the previously found V-Av molecules. ${ }^{6-13}$ This state is energetically favored in rather large mesoscopic samples, but it is never the ground state of the system. Consequently, more elaborate techniques are needed for its stabilization in experiment, such as an abrupt increase by the applied field (from negative to positive value) or an abrupt cooling of the symmetry-induced V-Av state. However, the very large $\Phi-T$ stability range of the second-generation $\mathrm{V}$-Av state, the very comprehensive size of the $\mathrm{V}$-Av molecule and the large variation in amplitudes of both superconducting order parameter and stray magnetic field inside the molecule (further enhanced for lower $\kappa$ and temperature) should all lead to facilitated future experimental observation of these predicted effects.

This work was supported by the Flemish Science Foundation (FWO-Vl), the Interuniversity Attraction Poles (IAP), Programme-Belgian State-Belgian Science Policy, ESFVORTEX program, and the ESF-AQDJJ network. *francois.peeters@ua.ac.be

${ }^{1}$ A. K. Geim, I. V. Grigorieva, S. V. Dubonos, J. G. S. Lok, J. C. Maan, A. E. Filippov, and F. M. Peeters, Nature (London) 390, 259 (1997); A. K. Geim, S. V. Dubonos, I. V. Grigorieva, K. S. Novoselov, F. M. Peeters, and V. A. Schweigert, ibid. 407, 55 (2000).

${ }^{2}$ V. V. Moshchalkov, L. Gielen, C. Strunk, R. Jonckheere, X. Qiu, C. Van Haesendonck, and Y. Bruynseraede, Nature (London) 373, 319 (1995).

${ }^{3}$ Y.-L. Lin and F. Nori, Phys. Rev. B 65, 214504 (2002); 50, 15953 (1994); Q. Niu and F. Nori, ibid. 39, 2134 (1989); F. Nori and Q. Niu, ibid. 37, 2364 (1988); R. Rammal, T. C. Lubensky, and G. Toulouse, ibid. 27, 2820 (1983).

${ }^{4}$ V. A. Schweigert, F. M. Peeters, and P. S. Deo, Phys. Rev. Lett. 81, 2783 (1998).

${ }^{5}$ A. Kanda, B. J. Baelus, F. M. Peeters, K. Kadowaki, and Y. Ootuka, Phys. Rev. Lett. 93, 257002 (2004).

${ }^{6}$ L. F. Chibotaru, A. Ceulemans, V. Bruyndoncx, and V. V. Moshchalkov, Nature (London) 408, 833 (2000).

${ }^{7}$ V. R. Misko, V. M. Fomin, J. T. Devreese, and V. V. Moshchalkov, Phys. Rev. Lett. 90, 147003 (2003).
${ }^{8}$ A. S. Mel'nikov, I. M. Nefedov, D. A. Ryzhov, I. A. Shereshevskii, V. M. Vinokur, and P. P. Vysheslavtsev, Phys. Rev. B 65, 140503(R) (2002).

${ }^{9}$ J. Bonča and V. V. Kabanov, Phys. Rev. B 65, 012509 (2001).

${ }^{10}$ B. J. Baelus and F. M. Peeters, Phys. Rev. B 65, 104515 (2002).

${ }^{11}$ C. Carballeira, V. V. Moshchalkov, L. F. Chibotaru, and A. Ceulemans, Phys. Rev. Lett. 95, 237003 (2005).

${ }^{12}$ R. Geurts, M. V. Milošević, and F. M. Peeters, Phys. Rev. Lett. 97, 137002 (2006).

${ }^{13}$ R. Geurts, M. V. Milošević, and F. M. Peeters, Phys. Rev. B 75, 184511 (2007).

${ }^{14}$ V. V. Khotkevych, M. V. Milošević, and S. J. Bending, Rev. Sci. Instrum. 79, 123708 (2008).

${ }^{15}$ M. V. Milošević, G. R. Berdiyorov, and F. M. Peeters, Appl. Phys. Lett. 91, 212501 (2007).

${ }^{16}$ G. R. Berdiyorov, B. J. Baelus, M. V. Milošević, and F. M. Peeters, Phys. Rev. B 68, 174521 (2003).

${ }^{17}$ J. Jurisson and R. J. Oakes, Phys. Lett. 2, 187 (1962); E. Coskun, Appl. Math. Comput. 106, 31 (1999).

${ }^{18}$ M. R. Connolly, M. V. Milošević, S. J. Bending, and T. Tamegai, Phys. Rev. B 78, 132501 (2008). 